\title{
Vortical field amplification and particle acceleration at rippled shocks
}

\author{
F. Fraschetti ${ }^{1}$ \\ Departments of Planetary Sciences and Astronomy, University of Arizona, Tucson, AZ, 85721, USA
}

\begin{abstract}
Supernova Remnants (SNRs) shocks are believed to accelerate charged particles and to generate strong turbulence in the post-shock flow. From high-energy observations in the past decade, a magnetic field at SNR shocks largely exceeding the shock-compressed interstellar field has been inferred. We outline how such a field amplification results from a small-scale dynamo process downstream of the shock, providing an explicit expression for the turbulence back-reaction to the fluid whirling. The spatial scale of the $X$-ray rims and the short time-variability can be obtained by using reasonable parameters for the interstellar turbulence. We show that such a vortical field saturation is faster than the acceleration time of the synchrotron emitting energetic electrons.
\end{abstract}

Keywords: $85-06$

\section{Introduction}

The origin of cosmic-rays (CRs) still eludes the theoreti- ${ }^{33}$ cal and observational efforts in astroparticle physics since their discovery more than a century ago. Space and ground-based ${ }_{36}$ experiments have been providing us with a wealth of multi- ${ }_{37}$ wavelength observations to identify the source and investigate ${ }_{38}^{37}$ the mechanism of acceleration in various energy bands. Individual shell-type Supernova Remnant (SNR) shocks accelerate ${ }_{40}$ charged particles and are believed to provide a significant fraction of the power sustaining the observed CR spectrum. Moreover, realistic corrugated shocks travelling in the inhomoge- ${ }_{43}$ neous interstellar space generate turbulence in the compressed post-shock fluid.

The inhomogeneity of the unshocked ISM observed over sev- ${ }^{45}$ eral scales [2] is expected to deform the shock surface rippling ${ }^{46}$ the initial local planarity up to scales many orders of magni- ${ }^{47}$ tude greater than the thermal ion inertial length. HST obser- ${ }^{48}$ vations of SN1006 [27] constrain the length-scale of the shock ${ }^{49}$ ripples to $10^{16}-10^{17} \mathrm{~cm}$. We focus on the interaction of a non- ${ }^{50}$ relativistic SNR rippled shock with the turbulence upstream of ${ }^{51}$ the shock, disregarding the contribution of accelerated particles ${ }^{52}$ at the shock, as justified later.

From detection of non-thermal $X$-ray rims [31,4], rapid time- ${ }_{54}$ scale variability of $X$-ray hot spots [30] and $\gamma$-ray emission in ${ }_{55}$ extended regions [1], a magnetic field at the shock far exceeding the theoretically predicted shock-compressed field has been ${ }_{57}$ inferred. Whether or not such a magnetic field amplification in ${ }_{58}$ SNR is to be associated with energetic particles at the shock is ${ }_{59}$ still subject of controversy.

Magnetic field amplification might be also relevant to in situ ${ }_{61}$ measurements of the plasma downstream of the solar-wind ter- ${ }_{62}$

${ }^{1}$ Associated Member to LUTh, Observatoire de Paris, CNRS-UMR8102 ${ }^{64}$ and Université Paris VII, 5 Place Jules Janssen, F-92195 Meudon Cédex, ${ }^{65}$ France. mination shock [8], where fluctuations have been measured of the same order as the mean, or to radio observations of $\mathrm{Mpc}$ scale shocks at the edge of galaxy clusters [7]. Strong magnetic fields are also required in Gamma-Ray Bursts (GRB) and Active Galactic Nuclei (AGN) outflows to enable sufficient production of non-thermal radiation. In the ISM magnetic energy density and thermal pressure are typically comparable and both amount to a fraction $10^{-9}-10^{-7}$ of the total internal energy density (including rest mass). Therefore, a compression by an even ultra-relativistic shock (bulk Lorentz factor 100), cannot produce the fraction $10^{-3}-10^{-1}$ predicted by GRB phenomenological models of afterglow light curves [25].

The passage of an oblique non-relativistic shock through inhomogeneous medium has been known for longtime to generate vorticity in the downstream flow [18]; in a conducting fluid the turbulent motion at scale $l$ with fluid velocity $v_{l}$ and local density $\rho$ leads exponentially fast to an amplified magnetic field $B^{2}=4 \pi \rho v_{l}^{2}[22]$. The encounter of a shock surface with a density clump, also called Richtmyer-Meshkov (RM) instability [6], has been also extensively investigated in plasma laboratory experiments (see [11] and references therein).

Recent numerical 2D-MHD simulations have shown that such an amplification can be very efficient [16, 17]. Ideal MHD applied to 2D rippled shocks has shown that the ISM turbulence might amplify exponentially fast the upstream magnetic field with a growth rate depending on shock and upstream medium properties [13]. Such an amplification is expected to occur downstream of the blast wave, regardless the presence of shockaccelerated particles. Magnetic field may also be enhanced by field line stretching due to Rayleigh-Taylor (RT) instability [19] at the interface between the ejecta and the interstellar medium, i.e., far downstream of the shock. In contrast with the vortical turbulence, late-time RT turbulence might be affected by the highest energy particle gyrating in the downstream fluid far from the shock [15]. However, RT structures are unlikely to 


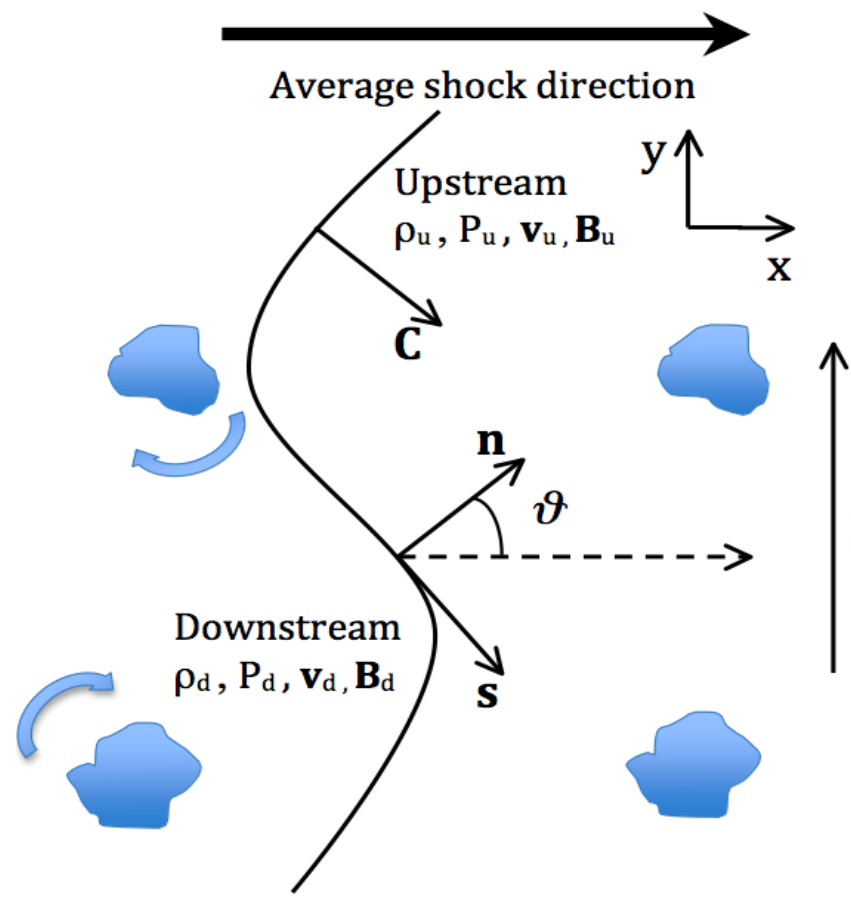

Figure 1: Encounter of a shock surface with density enhancement regions: for- 115 ward and lagging behind regions are formed that generate vorticity in the down-116 stream fluid. reach out the blast wave ([15] and references therein) and there-10 fore to interact with vortical turbulence. Thus the dynamo am-120 plification occurring locally behind the shock can be temporally ${ }^{121}$ and spatially disentangled from the field line stretching due to RT instability.

Two-dimensional simulations of relativistic shocks [23] show that small-scale dynamo can operate also downstream of ${ }^{123}$ the shocks with bulk Lorentz factor of a few unities. This sug-124 gests that the dynamo action downstream of shocks might shed ${ }^{125}$ light on the energy equipartition at magnetized shocks of AGN ${ }^{126}$ and Gamma-Ray Bursts.

\section{Macroscopic approach to rippled shock}

Constitutive equations - We consider the propagation of $\mathrm{a}^{13}$ 2D non-relativistic shock front in an inhomogeneous medium. ${ }^{132}$ Within the ideal MHD approximation, i.e., with no viscosity ${ }^{133}$ or heat conduction, the time evolution of the fluid velocity ${ }_{135}$ $\mathbf{v}=\mathbf{v}(x, y, t)$ and the magnetic field $\mathbf{B}=\mathbf{B}(x, y, t)$, is given, ${ }_{136}$ for infinitely conductive fluid, by

$$
\left\{\begin{array}{c}
\partial_{t} \mathbf{v}+(\mathbf{v} \cdot \nabla) \mathbf{v}+\frac{\nabla P}{\rho}+\frac{1}{4 \pi \rho}[\mathbf{B} \times(\nabla \times \mathbf{B})]=0 \\
\partial_{t} \mathbf{B}=\nabla \times(\mathbf{v} \times \mathbf{B})
\end{array}\right.
$$

where $\rho, P$ are respectively density and hydrodynamic pressure of the fluid (here $\partial_{t}=\partial / \partial t$ ). Note that the current density car-142 ried by CRs is here neglected: we aim to identify the growth ${ }_{143}$ of the magnetic energy as generated by the vortical motion of ${ }_{144}$ the background fluid only. Plasma heating by the shock might ${ }_{145}$ reduce the energy deposited in the magnetic turbulence and will ${ }_{146}$ be considered in a forthcoming publication.
Vorticity downstream of MHD shock - The vorticity shockgenerated is transported along the flow "frozen" into the fluid in the inviscid approximation (Helmholtz-Kelvin theorem). The medium upstream of the shock has $\omega=0$. The vorticity is calculated downstream at a distance from the shock large enough that the shock is infinitely thin, i.e., the thickness of the shock is much smaller than the local curvature radius at every point of the shock surface.

At a rippled shock the MHD Rankine-Hugoniot jump conditions cannot be applied globally as the directions normal and tangential vary along the shock surface. For a 2D shock propagating at average in the direction $x$ (all quantities are independent on $z$, see Fig 11, from the velocity field of the flow $\mathbf{v}=\left(v_{x}, v_{y}, 0\right)$, the vorticity is given by $|\omega|=|\nabla \times \mathbf{v}|=\omega_{z}$. We use a local natural coordinate system $(\hat{n}, \hat{s})$, where $\hat{n}=$ $(\cos \vartheta(t, s), \sin \vartheta(t, s))$ is the coordinate along the normal to the shock surface, $\hat{s}=(\sin \vartheta(t, s),-\cos \vartheta(t, s))$ is the coordinate parallel to the shock surface (Fig 1 ). We consider a seed-magnetic field upstream uniform and normal to the average direction of motion $\left(\mathbf{B}_{0}=\left(0, B_{0}^{y}, 0\right)\right.$, or $B_{n}=B_{0} \sin \vartheta$ and $B_{s}=-B_{0} \cos \vartheta$, see Fig 1 .

The turbulent field is assumed to be much greater than the shock-compressed field in the downstream flow, in agreement with observations, so that the amplification is efficient at the smallest scales (see Sect. 3). Thus, the vorticity produced downstream of a 2D shock propagating in an inhomogeneous medium with a uniform perpendicular upstream magnetic field (same as for parallel shock [13]) can be recast, neglecting obliqueness, in a simple form (we use $\partial_{x_{i}}=\partial / \partial_{x_{i}}$ ):

$$
\left|\delta \omega_{z}\right|=\frac{r-1}{r}\left[\left(\frac{C_{r}}{\rho}\right)_{u} \partial_{s} \rho+\partial_{s} C_{r}\right]-\frac{B_{n} \delta B_{s}}{4 \pi \rho C_{r}} \partial_{s} \vartheta,
$$

where $r=\rho_{d} / \rho_{u}$ is the compression ratio at the shock, $C_{r}$ is the shock speed relative to the upstream frame, $\delta B_{s}$ is the jump across the shock of the magnetic field in the direction locally tangential to the shock surface including the Rankine-Hugoniot compressed seed field and the turbulently amplified field and $B_{n}$ is the component in the direction locally normal to the shock surface including the unchanged Rankine-Hugoniot and the turbulent components.

Turbulent field amplification - The vortical turbulence described in the previous sub-section exponentially amplifies the total magnetic field. Since the amplification time-scale is of the order of the smallest eddies turnover time [3], the saturation occurs much faster at small-scale [20]. This is the key feature of the small-scale dynamo. The unperturbed field is initially too weak to affect the fluid velocity field and the turbulent field grows exponentially fast, until the magnetic energy produces non-negligible effects on the velocity field and then saturates.

The small-scale dynamo theory predicts that the turbulent field obeys an unbounded exponential amplification at a rate $\beta$ [20, 21]: $d \varepsilon / d t=2 \beta \varepsilon$, where $\varepsilon=B^{2} / 8 \pi \rho$ is the total magnetic energy per unit of mass, including seed and turbulent fields. As shown in [20], the isotropy and homogeneity of the fluid velocity correlation entails the following simple relation between the amplification rate of $\varepsilon$ and the vorticity generated downstream of the shock: $\beta \simeq(\pi / 3) \delta \omega_{z}$. 


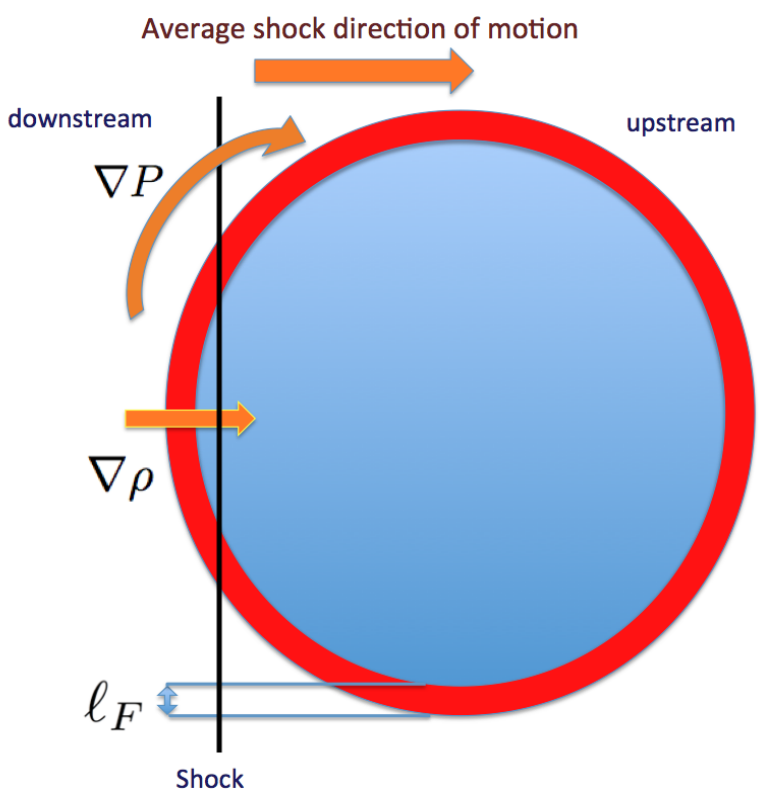

175

Figure 2: Generation of the baroclinic term of the vorticity at the shock crossing 176 in the condensation layer of thickness $\ell_{F}$.
If we recast Eq.(2) as $\left|\delta \omega_{z}\right|=(3 / \pi)\left(\tau^{-1}-\alpha \varepsilon\right)$, then $\varepsilon$ satisfies $^{179}$

$$
\frac{d \varepsilon}{d t}=2\left(\tau^{-1}-\alpha \varepsilon\right) \varepsilon
$$

where $\tau^{-1}=\frac{\pi}{3} \frac{r-1}{r}\left[\left(C_{r} / \rho\right)_{u} \partial_{s} \rho+\partial_{s} C_{r}\right]$ is the local growth rate ${ }^{183}$ of $\varepsilon$ and $\alpha=(2 \pi / 3) \partial_{s} \vartheta / C_{r}$ is the local back-reaction; the initial ${ }^{184}$ condition for Eq. 3 is $\varepsilon(0)=\varepsilon_{0}=v_{A}^{2} / 2=B_{0}^{2} / 8 \pi \rho$. In Eq. 3$\}^{185}$ we have assumed that the turbulence dominates over $B_{0}$, i.e., $\delta B_{s} / \sqrt{8 \pi \rho} \sim \sqrt{\varepsilon}$ and $B_{n} / \sqrt{8 \pi \rho} \sim \sqrt{\varepsilon}$ : the turbulence grows ${ }^{187}$ isotropically downstream at the shock curvature scale as a con- ${ }^{188}$ sequence of the isotropy of the flow velocity field [20].

Neglecting the time dependence of $\tau$ (the magnetic modes ${ }^{190}$ grow slowly for initially weak field [20]), the solution is readily ${ }^{191}$ found:

$$
\frac{\varepsilon}{\varepsilon_{0}}(t)=\left(\frac{B}{B_{0}}\right)^{2}(t)=\frac{e^{2 t / \tau}}{1-\alpha \tau\left(1-e^{2 t / \tau}\right) v_{A}^{2} / 2},
$$

for a uniform average interstellar matter density.

\section{Comparison with multiwavelength SNR observations}

The growth rate of $\delta B$ can be approximated as $\tau^{-1} \sim C_{r}\left(R_{C}+{ }^{199}\right.$ $\left.\ell_{F}\right) /\left(R_{c} \ell_{F}\right)$, where $R_{c}$ is the local curvature radius of the shock 200 surface. Thus $\tau^{-1}$ increases with shock speed and it depends201 mainly on hydrodynamic quantities. If $\ell_{F} \ll R_{c}$, it holds $\tau \sim_{202}$ $\ell_{F} / C_{r}$ : the amplification saturates faster for smaller $\ell_{F}$.

As the magnetic field strengthens, it reacts to field lines204 whirling halting the turbulence growth. In more general terms, 205 as the field increases by dynamo action it also releases its ten-206 sion by unwinding at a rate of order of Alfvén speed: the back-207 reaction grows with the turbulent field Alfvén speed [20]. The 208 local back-reaction of the field $\alpha \sim \partial_{s} \vartheta / C_{r}$ can be estimated by209 $\alpha \sim \vartheta /\left(R_{c} C_{r}\right)$.

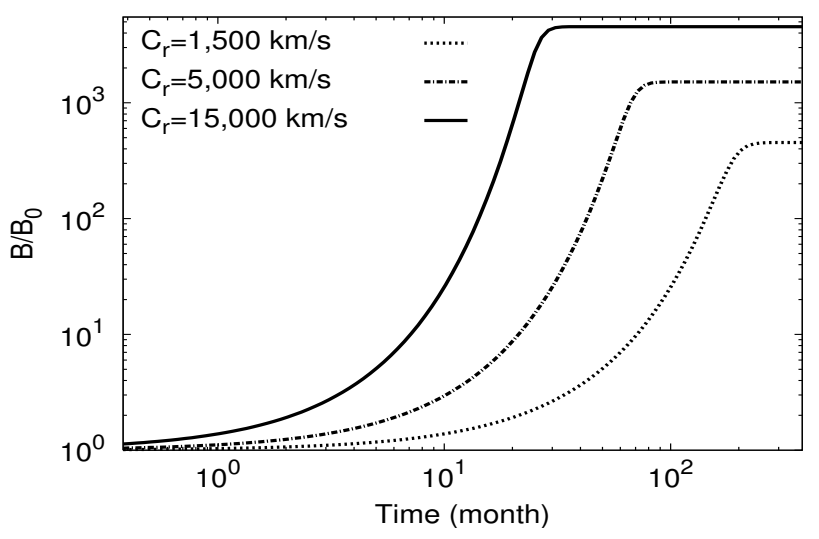

Figure 3: Saturation of the total magnetic field for various shock speed $C_{r}$ is shown: $C_{r}=1,500 \mathrm{~km} / \mathrm{s}, C_{r}=5,000 \mathrm{~km} / \mathrm{s}, C_{r}=15,000 \mathrm{~km} / \mathrm{s}$, assuming $R_{C}=10^{17} \mathrm{~cm}$ and $\ell_{F}=10^{16} \mathrm{~cm}$, that results in $\tau \lesssim \ell_{F} / C_{r} \sim 3$ years for $C_{r} \gtrsim 5,000 \mathrm{~km} / \mathrm{s}\left(\vartheta=0.1 \mathrm{rad}, r=4\right.$ and $\left.v_{A}=10^{-4} c\right)$.

Fig 3 depicts the growth of the turbulent field for various shock speeds, assumed constant in time: given an ISM field of the order of $B_{0} \sim 3 \mu \mathrm{G}$, the turbulent field saturates at $B \sim 1.2-3 . \mathrm{mG}$ for $C_{r}=1,500-5,000 \mathrm{~km} / \mathrm{s}$ on the year time-scale. Such a rapid growth of magnetic energy is compatible with $X$-ray observations of SNRs $R X J 1713.7$ - 3946 $\left(C_{r}<4,500 \mathrm{~km} / \mathrm{s}\right.$ [30]) and Cas A [26] brightness variations detected on year time-scale in small-scale hot spots structures, attributed to synchrotron electron cooling. Using $R_{c}=10^{17}$ $\mathrm{cm}$ and $\ell_{F}=10^{16} \mathrm{~cm}$, we find an amplification to $B \sim 3 . \mathrm{mG}$ within 3 years. Such a value of $\ell_{F}$ is to be compared with the spatial scale of the Chandra RXJ1713.7 - 3946 bright spots, estimated as $\lesssim 0,03$ pc. Similar length $\left(\sim 10^{14}-10^{16} \mathrm{~cm}\right)$ and time $(\sim 1 \mathrm{yr})$ scales are found in simulations of the effects of magnetic field turbulence on the observed synchrotron emission images and spectra in SNRs [9]. Thus, the magnetic energy increase and the $X$-ray variability might have a time-scale ( $\sim 1 \mathrm{yr}$ ) much lower than the SNR hydrodynamic time-scale and might occur in middle-aged, not necessarily young, SNRs ( $R X J 1713.7$ - 3946 age is estimated as 1,600 yr [29]). The high shock speed $C_{r} \sim 15,000 \mathrm{~km} / \mathrm{s}$ in Fig 3 is comparable to observations of the youngest SNR in our galaxy, i.e., 100 years old G1.9 + 0.3 [28]. Thus, a rapid field saturation even up to $B \sim 10 \mathrm{mG}$ is predicted at SNR shocks within a few months.

\section{Constraints on particle acceleration}

If the thickness of the density steepening layer at the boundary of the ISM density clumps is identified as the Field length $\ell_{F}$, for typical cold ISM, we can use $\ell_{F} \simeq 3.3 \times 10^{16} \mathrm{~cm}$, with an uncertainty depending on ionization and heating/cooling properties [5]. Such an $\ell_{F}$ is compatible with the ripple scale inferred by optical observations [27]. Thus, for a typical middleaged SNR with shock speed $C_{r} \sim 5,000 \mathrm{~km} / \mathrm{s}$, the growth timescale of the vortical turbulence is $\tau \simeq \ell_{F} / C_{r} \simeq 6.7 \times 10^{7} \mathrm{~s} \sim 1.9$ yrs.

A simple argument shows that $\tau$ is shorter than the typical acceleration time-scale for energetic electrons at the shock, i.e., 
$\tau_{a c c}$. Modulo a factor of order of unity, $\tau_{a c c} \simeq \kappa_{E} / C_{r}^{2}$, where the 265 diffusion coefficient $\kappa_{E}$ (neglecting its change across the shock) ${ }^{266}$ depends on the particle energy and on the magnetic field orien ${ }^{267}$ tation. If the seed magnetic field is parallel to the local shock ${ }_{269}^{268}$ normal, the diffusion coefficient governing the electron accel-270 eration $\kappa_{E}$ is necessarily greater than the Bohm diffusion coef-271 ficient $\kappa_{B}$, corresponding to $\lambda \simeq r_{g}$, where $\lambda$ is the mean free ${ }_{273}^{272}$ path of the charged particle and $r_{g}=p c / e B$ is the particle gyro- ${ }_{274}$ radius. The typical energy of an electron emitting synchrotron 275 radiation at $5 \mathrm{keV}$ in an amplified magnetic field $B \sim 100 \mu \mathrm{G}^{276}$ is $E \sim 50 \mathrm{TeV}$. Thus, for an energetic electron diffusing at the ${ }_{278}^{277}$ shock in the Bohm regime, $\kappa_{E}=r_{g} c / 3 \simeq 3.3 \times 10^{23} E_{13} / B_{2279}{ }^{278}$ $\mathrm{cm}^{2} / \mathrm{s}$, where $E_{13}$ is the electron energy in units of $10 \mathrm{TeV}$ and 280 $B_{2}$ the magnetic field in the $X$-ray rim in units of $100 \mu \mathrm{G}$. Thus, ${ }_{282}^{281}$ we obtain $\tau \simeq \kappa_{E} / C_{r}^{2}=1.9$ years $\simeq \tau$.

Bohm diffusion, despite largely used in the literature because 284 of the lack of self-consistent diffusion theory in strong turbu-285 lence, describes transport only for a very limited range of par- $^{286}$ ticle energy (see [10, 14]). Since the scattering diffusion coeffi ${ }^{287}$ cient $\kappa_{E}$ in most cases is much greater than $\kappa_{B}$, the inferred field 289 amplification might occur on a time-scale much shorter than ${ }^{290}$ acceleration time-scale of particles scattering back and forth ${ }_{292}^{291}$ across the shock. Our simple estimate, derived from the $X-$ ray $_{293} 292$ synchrotron parameters and the inferred strong field, holds re-294 gardless the location of the emitting region, whether upstream ${ }^{295}$ or downstream of the shock. The change of the structure of ${ }_{297}^{296}$ the turbulence across the shock, due to the anisotropic shock ${ }_{298}$ compression and the vortical amplification downstream shown299 here, is not expected to modify significantly our estimate of ${ }^{300}$ $\tau_{a c c}$

\section{Conclusion}

By applying first principles to a $2 \mathrm{D}$ rippled shock, we have outlined the derivation of temporal evolution and saturation of the turbulent magnetic field downstream of the shock, including the non-linear field back-reaction. We conclude that the saturation of $B$ by small-scale dynamo action depends on the shock speed, on the thickness of the density steepening layer at the boundary of the ISM density clumps and on the shock curvature radius, but not on the size of the ISM clumps. Our finding shows that small-scale dynamo might explain non-thermal $X$ ray observations and agrees with the optical upper limit on the scale of shock ripples. The magnetic field enhancement described here occurs faster than acceleration time-scale of synchrotron emitting energetic electrons.

Acknowledgment - The author thanks ISSI for providing stimulating environment. The support from NASA through the Grants NNX10AF24G and NNX11AO64G is gratefully acknowledged.

\section{References}

[1] Acciari, V. A. et al. 2011, ApJ Lett., 730, 20

[2] Armstrong, J. W., Rickett, B. J. and Spangler, S. R. 1995, ApJ 443, 209

[3] Balsara, D. S. and Kim, J. 2005, ApJ, 634, 390

[4] Bamba, A., Yamazaki, R., Ueno, M., Koyama, K. 2004, Adv. Space Res. 33,376
[5] Begelman, M. C. and McKee, C. F. 1990, ApJ, 358, 375

[6] Brouillette, M. 2002, Annu. Rev. Fluid Mech, 34, 445

[7] Brüggen, M., Bykov, A. M., Ryu, D. and Röttgering, H. 2012, Space Science Reviews, 166, 187

[8] Burlaga, L. F., Ness N. J. and Acuña, M.H. 2007, ApJ, 668, 1246

[9] Bykov, A. M., Uvarov, Y. A. and Ellison, D. C. 2008, ApJ Lett., 689, 133

[10] Casse, F., Lemoine, M. and Pelletier, G. 2002, Physical Review D, 65, 023002

[11] Dimonte, G. and Ramaprabhu, P. 2010, Phys. of Fluids, 22, 014104

[12] Field, G. B. 1965, ApJ. 142, 531

[13] Fraschetti, F. 2013, ApJ, 770, 84

[14] Fraschetti, F. \& Giacalone, J. 2012, ApJ, 755, 114

[15] Fraschetti, F., Teyssier, R., Ballet, J. and Decourchelle, A. 2010, A\&A, 515, A104

[16] Giacalone, J. and Jokipii, J.R. 2007, ApJ Lett., 663, 41

[17] Inoue, T., Yamazaki, R., Inutsuka, S. and Fukui, Y. 2012, ApJ, 744, 71

[18] Ishizuka, T., Hashimoto, Y. and Ono, Y. 1964, Progress of Theoretical Physics 32, 207

[19] Jun, B. I. , Norman, M. L. and Stone, J. M. 1995, ApJ, 453, 332

[20] Kulsrud, R.M. Plasma Physics for Astrophysics (Princeton University Press, 2005), Chap. 13

[21] Kulsrud, R.M. and Anderson, S.W. 1992, ApJ, 396, 606

[22] Landau, L. D. and Lifshitz, E. M. Electrodynamics of continuous media (Pergamon Press, 1960)

[23] Mizuno, Y., Pohl, M., Niemiec, J. et al. 2011, ApJ, 726, 62

[24] Noutsos, A. 2012, Space Science Reviews, 166, 307

[25] Panaitescu, A. 2005, Monthly Not. Royal Astron. Soc., 363, 1409

[26] Patnaude, D. J. and Fesen, R. A. 2009, ApJ, 697, 535

[27] Raymond, J. C., Korreck, K. E., Sedlacek, Q. C., Blair, W. P., Ghavamian, P. and Sankrit, R. 2007, ApJ, 659, 1257

[28] Reynolds, S. P., Borkowski, K. J., Green, D. A., Hwang, U., Harrus, I. and Petre, R. 2008, ApJ Lett., 680, 41

[29] Stephenson, F. R. \& Green, D. A. 2002, Historical Supernovae and their remnants, Oxford Univ. Press

[30] Uchiyama, Y., Aharonian, F. A., Tanaka, T., Takahashi, T. and Maeda, Y. 2007, Nature 449, 576

[31] Vink, J. and Laming, J. M. 2003, ApJ, 584, 758 